\title{
SIMULATION OF A SIGNAL QUALITY SURVEY
}

\author{
Douglas J. Morrice \\ MSIS Department \\ CBA 5.202 \\ The University of Texas at Austin \\ Austin, Texas 78712-1175
}

Herb Schwetman

Mesquite Software, Inc.

4210 Spicewood Springs Rd., \#201

Austin, TX 78759
Peter W. Mullarkey

End-to-End Simulation Department 8311 North RR 620

Schlumberger Austin Product Center Austin, Texas 78726
Astrid S. Kenyon

Department of ORIE

ETC 5.110

The University of Texas at Austin

Austin, TX 78712-1175

Jingfang Zhou

End-to-End Simulation Department

8311 North RR 620

Schlumberger Austin Product Center

Austin, Texas 78726

\begin{abstract}
This paper describes a simulator for a large outdoor operation called a signal quality survey. Design and implementation of the simulator follows an object oriented approach with a primary focus of modeling operations cycles. The simulator is implemented in Visual $\mathrm{C}++/$ CSIM17. Excel acts as the primary user interface. A Microsoft Foundations Classes Single Document Interface application acts as a secondary user interface for simulation animation. We discuss the benefits of using discrete event simulation for this application. We also report on some of the challenges encountered during the implementation stages of the project.
\end{abstract}

\section{INTRODUCTION}

In this paper, we describe the design and implementation of a simulator for a large outdoor operation called a signal quality survey. Signal quality surveys are conducted over large geographical areas (tens to hundreds of square kilometers). They are projects taking anywhere from a few days to a few years with crews ranging from 20 to 1000 people, requiring capital equipment valued in the tens of millions of dollars, and generating survey revenues ranging from tens to hundreds of millions of dollars. The main challenge is to bid on, plan for and conduct these large, complicated, and expensive projects in a profitable manner.

The primary objective associated with modeling signal quality survey is to characterize the various loosely coupled, interacting operations in a realistic manner. Achieving this objective is complicated by several factors: terrain, equipment failures, wildlife (causes damage to equipment), weather, permitting (i.e., permission to conduct the survey on public or private property), personnel problems, community relations, cultural factors, environmental and legal restrictions. Given the complexity of operations and the uncertainty associated with several of these factors, simulation was identified as an important decision support tool for use in understanding, quantifying, and improving signal quality survey operations.

The remainder of the paper is organized in the following manner. Section 2 contains a description of the problem. Section 3 describes a conceptual model used in the design of the simulation. Section 4 describes the implementation of the simulation model. Section 5 discusses the benefits of simulation in this application. Section 6 contains a discussion on some of the challenges encountered during the implementation of the simulation.

\section{PROBLEM DESCRIPTION}

Signal generation and signal monitoring (receiving) constitute the two main operations in a signal quality survey. Locations from which a signal is sent are called source locations and locations at which a signal is received are called receiver locations. The source and receiver locations form a geometric pattern over a large geographic area. For example, Figure 2.1 depicts a survey design with sources arranged along several parallel lines (source lines) and receivers laid out along parallel lines (receiver lines) perpendicular to the source lines. Along each source and receiver line, locations are typically spaced about 50 meters apart. The entire survey area can range from tens to hundreds of square kilometers. 


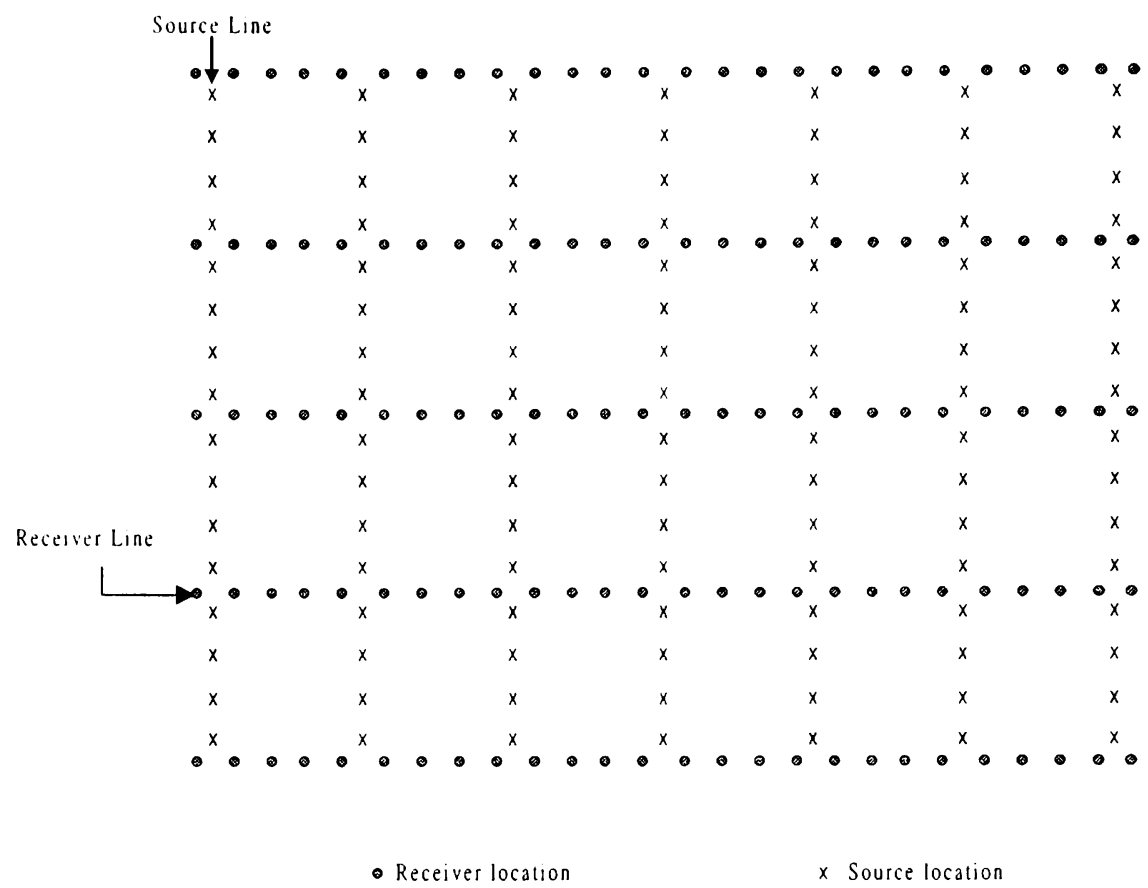

Figure 2.1: Signal Quality Survey

To conduct the survey, signals are sent by a signal crew from a sequence of source locations (one-at-atime) to a sequence of overlapping receiver location sets. Figure 2.2 depicts two source locations along with their receiver location sets. Each set is enclosed in a rectangle centered on its corresponding source location. Source sequencing is a function of the design of the survey and user input on the partitioning of the survey into subsections. Surveys are partitioned because of receiving equipment constraints and physical and cultural obstacles on the survey. The signals are monitored by equipment at a set of receiver locations and then recorded by an acquisition system operated by a recording crew. Measurements from a single source location take less than a minute. Moving equipment from one receiver location set to the next can take several minutes or hours. To avoid signal sending delays, extra equipment is used to cover several receiver location sets ahead of the set required by the current source location. The acquisition system turns different receivers on and off so that the required receiver set is turned on for each source measurement.

Typically, there is not enough receiver equipment available to cover the entire survey area, so that equipment movement is needed. Receiver equipment consists of sensors, batteries to power the equipment, and cables to connect all the equipment together. The equipment is brought to location by transport vehicles. It is then installed (unpacked, laid out, and connected) by crews of workers called layout crews. The transport vehicles retrieve equipment from other parts of the survey where it is no longer required. However, before doing so, the equipment must be prepared (disconnected, rolled up, and packaged) by crews of workers called packing crews. The movement of receiver equipment is the most time and resource intensive operation on a signal quality survey. The goal of this operation is to move receiving equipment in a manner that does not impede signal generation production.

A signal quality survey contains a lot of equipment that needs to be kept in proper working order. Crews called trouble shooters are assigned to test and repair failed receiving equipment. They also perform other types of routine maintenance on the receiving equipment such as battery charging and replacement. Maintenance of other equipment such as transport vehicles is handled by mechanics personnel.

The overall operation of a signal quality survey involves the coordination between signal generation, recording, transport, layout, packing, and maintenance. 


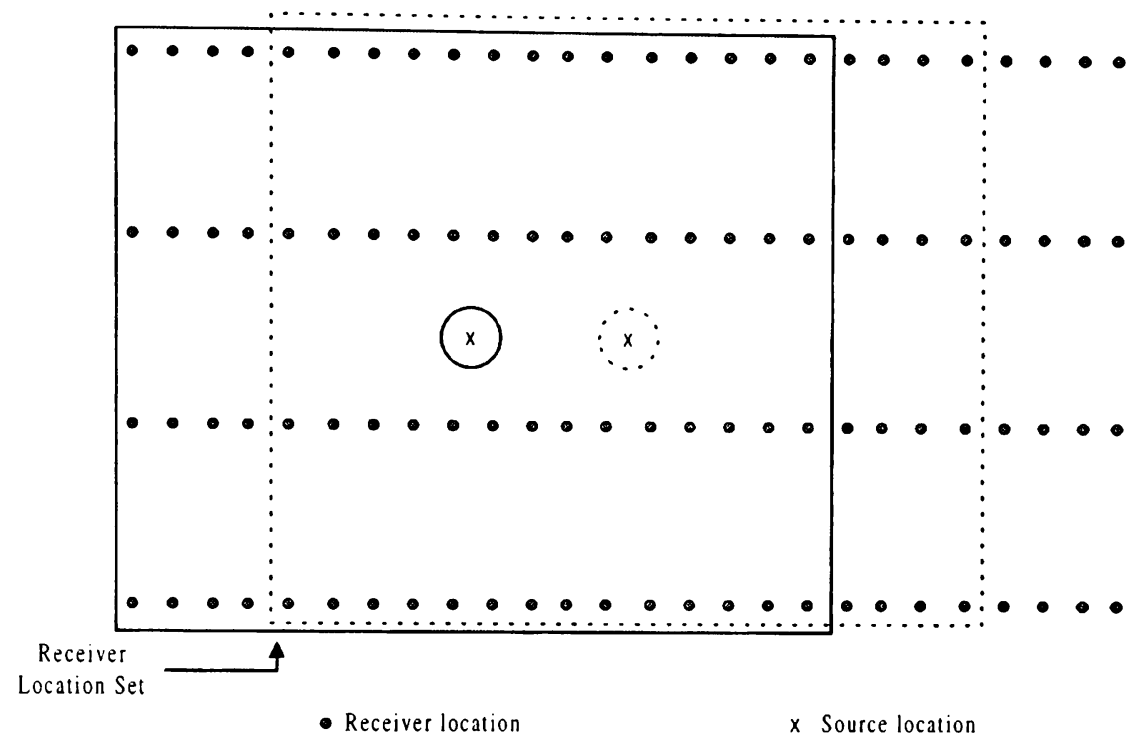

Figure 2.2: Source and Receiver Relationships

\section{CONCEPTUAL MODEL}

We took an object oriented approach to design the simulation model (Joines and Roberts 1994). The objects fall into three main categories:

i. Survey design or geometry.

ii. Resources to conduct the survey operations.

iii. Managers to coordinate the resources on a specified survey geometry.

The focus of this section will be the resource objects because they are dynamic objects (i.e., objects that perform operations in which simulated time elapses) that constitute the heart of the simulation model. Objects in categories (i) and (iii) are not dynamic but instead contain information on survey design and operations strategies. They will be discussed in more detail in the next section.

The main survey operations objects include:

i. transport vehicles,

ii. layout crews,

iii. recording crew,

iv. signal crews,

v. packing crews,

vi. trouble shooting crews, vii. mechanics personnel.

Each object contains one or more methods to model the activities (or operations) performed by the object in the simulation. In order to design these methods as well as to model interactions between the various objects, we identified operations cycles at three levels:

i. for the entire duration of the survey,

ii. daily,

iii. for the objects involved in operations (e.g., transport vehicles, crews), both individually and collectively.

The survey duration operations cycle includes three phases: mobilization, signal generation and recording, and demobilization. At the beginning of a survey, all equipment is delivered to a base of operations camp (or base camp). Mobilization entails moving equipment from the base camp location to the field. Signal generation and recording is considered production time where signals are being sent and received. It also involves the movement of equipment to support these operations. Demobilization involves moving all equipment back to the base camp when it is no longer required on the field. It is important to note that the phases are usually overlapping. For example, it is generally the case that 
excess equipment is available for recording. Therefore, signal generation and recording may begin before all equipment has been moved out of the base camp, i.e. before the mobilization phase ends.

It is advantageous to explicitly consider the survey operations cycle hecause, in each phase, an object may be expected to perform different tasks or to behave according to different decision rules. For example, during signal generation and recording, a transport vehicle retrieves the minimum of how much equipment is required on a particular part of the survey and its capacity. During the demobilization phase the transport vehicle always retrieves its capacity in order to return equipment to the base camp.

A daily operation cycle exists because on most surveys operations cease at night. The daily operation includes morning startup, production, and evening shutdown. Startup includes such things as travel to the survey, turning on and testing the equipment, and diagnosing and repairing problems. Production includes all activities directly associated with signal generation and recording. Shutdown involves such things as preparing equipment for shutdown, refueling, logging data, and traveling back to base camp from the survey. Accurate modeling of the daily operations cycle is crucial because startup and shutdown (considered nonproductive operating hours) can account for a significant portion of the total operating hours each day.

At the lowest level, each object on a signal quality survey has its own operations cycle. For example, an operations cycle for a transport vehicle is shown in Figure 3.1. The cycle starts with the transport vehicle requesting a work assignment (or notifying a manager that it is available for work). When work becomes available, the transport vehicle is given an assignment. The assignment entails pickup of equipment on one part of the survey and dropoff at another part. When the transport vehicle is free, another work request is made and the cycle repeats. Figure 3.1 depicts the most basic form of an operations cycle for the transport vehicle. It could be made more complicated (and realistic) by including scheduled maintenance (e.g., oil check, refueling) and unscheduled breakdowns and repairs.

Objects do not act in isolation on a signal quality survey. Rather they act in concert to form a larger operations cycle. For example, the objects that directly support shooting and recording form a larger operation cycle with the following five steps:

i. transport vehicles pickup equipment prepared by the packing crews and drop off receiver equipment for installation by the layout crews.

ii. layout crews install receiver equipment for eventual use by the recording crew.

iii. a recording crew controls the sending of signals by the signal crews and records signals at a recording station.

iv. signal crews send signals which are captured by an installed set of receiving equipment. After each signal, equipment no longer required at a receiving location is made available to the packing crews for pickup preparation.

v. packing crews prepare available equipment for pickup by the transport vehicles.

Figure 3.2 summarizes the above sequence in an operations cycle diagram. The diagram depicts that each object cycles through its own local operations steps and forms a step in the larger operations cycle which represents the progression of the signal quality survey.

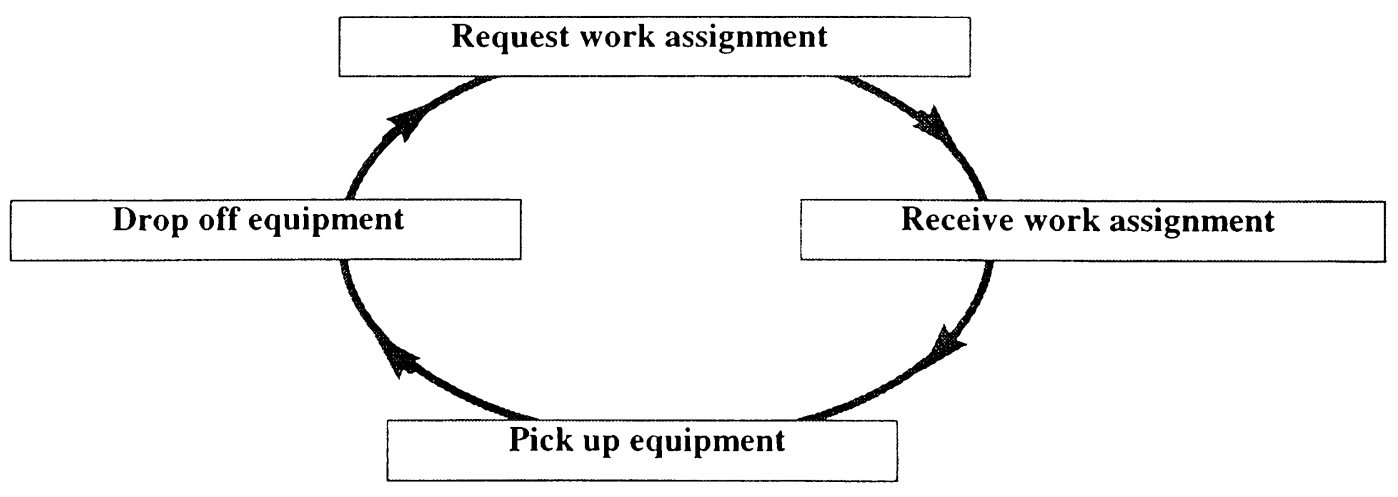

Figure 3.1: The Operations Cycle for a Transport Vehicle 
Again, it is important to note that Figure 3.2 depicts the most basic operations cycle for the five objects that directly support signal generation and recording on the survey. It could be made more realistic by adding equipment failures and repairs and other contingency plans when uncertainties arise such as a change in the weather. Additionally, the cycle could also include two additional objects: trouble shooters and mechanics personnel. Although the latter are not directly involved in signal generation and recording, equipment failures can halt production. Therefore, in the simulation model, the operations cycle in Figure 3.2 is embellished to include equipment failures and repairs along with trouble shooters and mechanics personnel.

Modeling operations cycles at the object level facilitates the design, implementation, refinement, and evolution of the simulation model. For each object, the operations cycle model provides the basis for the method(s) that control(s) the object's behavior during the simulation. The larger operations cycle model formed by the objects working in concert (e.g., Figure 3.2) provides the basis for the core of the simulation model.

\section{IMPLEMENTATION}

This section will discuss the software architecture used to organize the core simulator, the components used to implement that architecture and how they fit together, and the development tools used.

\subsection{System Architecture}

The overall architecture of the simulation model can be viewed as an example of the pipe and filter model (as described in Shaw and Garlan 1996). The basics of this design are that each operation or task is viewed as a filter that gets its input from an input stream and writes its output to an output stream. In this specific case the input stream is usually an ordered queue of receiver hardware and the output stream is an "order on entry" queue of the same type. One key aspect of this architecture is that it makes adding new operations or tasks reasonable, since as long as they know how to read from an ordered queue and write to another ordered queue, integration is quite simple and focused.

\subsection{Components and Relations}

There may be several ways to view the composition of the simulation model. One way is to consider the simulation as being composed of three types of objects: geometry - ones that represent the basic geometric information about the survey (e.g., receiver and sources locations and lines, size of active area); resources - ones that relate to the resources available to do the survey (e.g., number of vehicles, number of crews); and finally the managers - ones that use the available resources to act on the specified geometry to get the survey done.

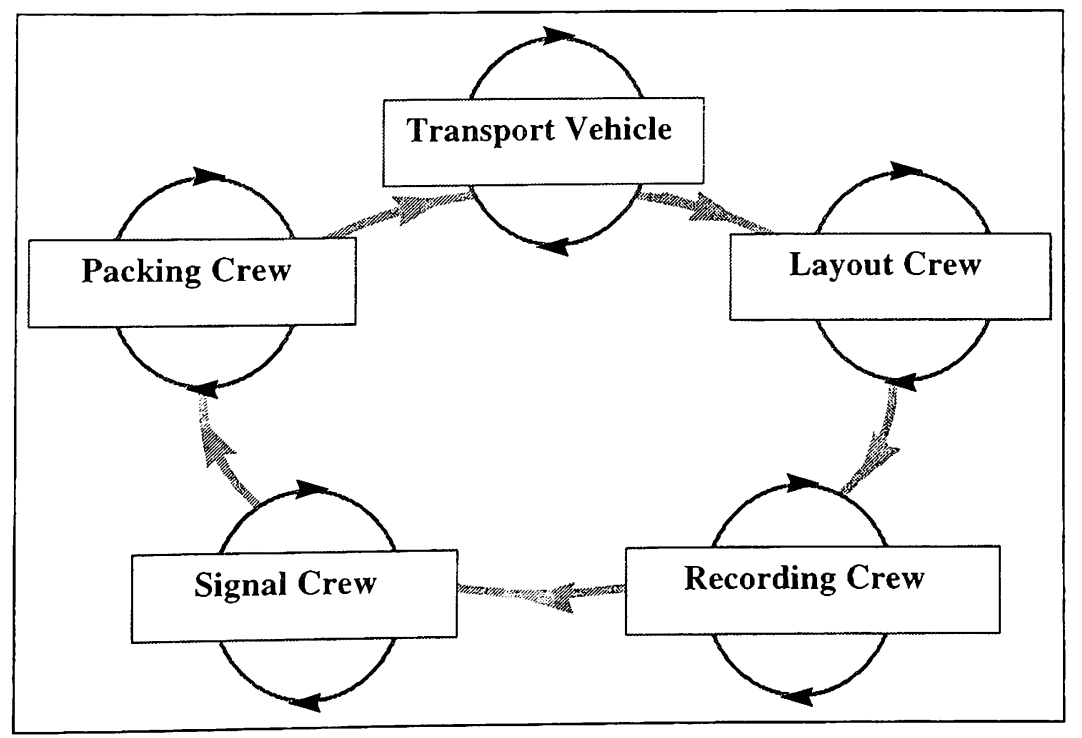

Figure 3.2: A Cycle for Shooting and Recording Operations 
The data in the geometry classes are considered the fixed infrastructure on which the simulation is performed (i.e., these data are not subject to change during the execution of a simulation). Survey designs are not generated by the simulator but by separate off-the-shelf software packages. This software generates a design and writes it using an industry standard format to a data file that is read by the simulator.

The group of classes that embody the link between the survey geometry and the actual project resources can all be thought of as Managers. Their basic role is to coordinate their task by looking for an available crew and the resources that crew needs to do their task, and fusing those two together to achieve that task. As an example, the PackingManager looks at the ordered queue of equipment to be packed, and when it gets to a certain level, looks for an available crew that can do Packing, and dispatches that crew to handle that set of equipment. This same idea of looking at two queues, and dispatching tasks is common in the packing manager, vehicle manager, layout manager, and recording manager. It should be noted that the order in which source locations are visited is not a dynamic or managed aspect of the simulator. This overall processing order is based solely on geometric information (the survey design and optional user partitioning of the survey).

In our implementation of the simulation model, we attempted to separate major policies (or strategies) from the mechanism of the simulation model.

\subsection{Overview of Simulation Components and Development Environment}

Figure 4.1 depicts the architecture of the simulator. The simulator runs on a PC platform in Windows 95 since this is the platform of choice for field personnel. Excel constitutes the main user interface because field personnel are currently using Excel to support their operations. In fact, much of the data required to drive the simulator already exists in Excel spreadsheets. The user is able to set all simulation parameters and run controls, and invokes the simulator from Excel.

The simulator running behind Excel was implemented in Visual $\mathrm{C}++$ in the Microsoft Development Environment as a Microsoft Foundations Classes (MFC) Application (Microsoft Corporation 1995). In addition, CSIM17, a library of $\mathrm{C}++$ routines for discrete event simulation, constitutes the simulation engine (Mesquite Software, Inc. 1994). When the simulator is invoked, it reads data from Excel and information on the survey design from the survey data file.

CSIM17 provides the core functionality for a process interaction approach to discrete event simulation. CSIM 17 was used because as a library of $\mathrm{C}++$ classes and methods it could be integrated into our overall application and by its component-nature, it is flexible. The main features that were used include support for processes, and events and messages to coordinate and communicate between processes. Additionally, CSIM17 is fast, which is an important feature for simulating iarge surveys. A simulation environment might have been selected but none were found that supported a problem of this type (open field logistics) and scale.

The animation interface is a MFC Single Document Interface (SDI) application. It displays the progress of the simulation from a bird's eye view of the survey. It also displays other information on the simulation in the form of messages and statistics. Once the simulation is complete, the animation interface permits the user to save the simulation results back to Excel. Additionally, the user can load new parameter values from the spreadsheet for subsequent simulation runs.

Once the simulation results are back in Excel, the user can use this information to embellish other models already resident in the spreadsheet. In addition, the data is available for analysis using the extensive statistical and graphics capabilities available in Excel.

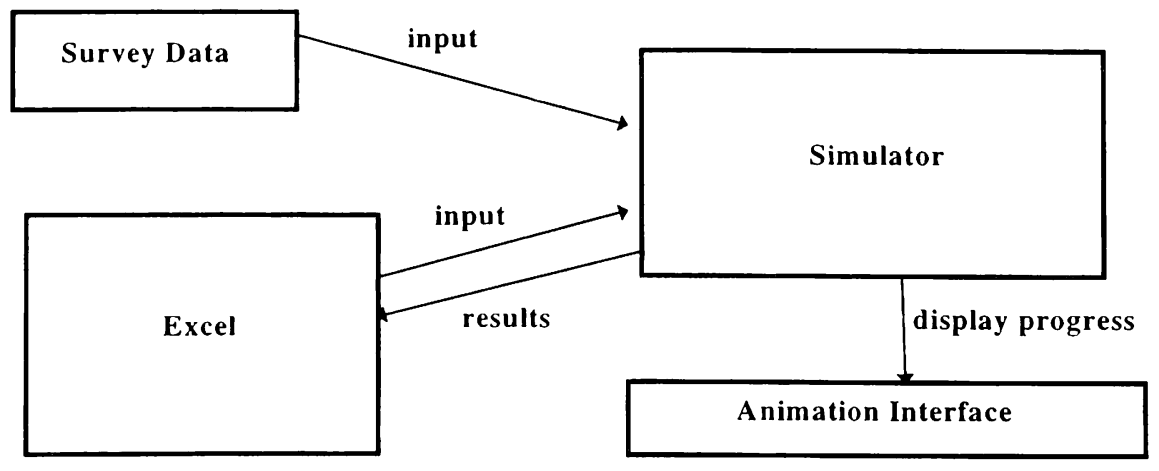

Figure 4.1: Simulation Components and Data Connections 


\section{BENEFITS}

The simulation model improves the planning and operations of a signal quality survey in a number of ways. In particular, simulation:

1. provides a more accurate quantification of survey duration times than the current modeling approaches. Current approaches include spreadsheets (primary) and project planning tools (secondary). Neither methodology can accurately model the complex operational details of a signal quality survey like simulation. Therefore, a properly designed and calibrated simulation will provide improved estimates of the duration of surveys. Better estimates directly impact the bottom line by enabling a decision maker to construct more accurate bids for a survey.

2. provides a better way to account for uncertainty. Current spreadsheet models use point estimates to quantify operational tasks such as signal crew movement times. The uncertainty associated with these tasks is incorporated into aggregate correction factors (e.g., terrain adjustment factor) which are used to adjust the spreadsheet model results. With simulation, uncertainty in such things as crew and vehicle movement times, and equipment failures and repairs can be directly incorporated into the model using probability distributions. The latter is a much more accurate way to account for uncertainty. Overall, simulation will provide a better way for decision makers to quantify and manage risk.

3. permits what-if or scenario analysis. Simulation will create new opportunities for experimenting with different design and operating strategies. This includes consideration of different types of survey designs, different source sequencing strategies, different resource allocation strategies, and different decision rules. Based on points (1) and (2), spreadsheets models cannot handle this task. Additionally, experimentation in the ficld is too costly.

4. permits the examination of complex interaction effects from simultaneously changing policies or input parameters (both will be referred to as factors). Experienced field personnel might be able to predict the effect of changing a single factor (e.g., an increase in the number of transport vehicles). However, it is much more difficult, even for experienced personnel, to predict the effect of changing multiple factors simultaneously (e.g., increase equipment, vehicles, and crews simultaneously). Simulation can be used to explore and quantify the benefits of such changes.

\section{CHALLENGES}

From this start, this project has received a tremendous amount of management support. In total, project personnel were able to visit around ten survey sites. Historical data from several surveys were sent from the ficld. Additionally, about two dozen field operations and enginecring personnel provided input used to design and test the simulator. Finally, some key field personnei participated in an in-house course on discrete event simulation in the summer of 1996 in order to better understand the technology being used in the simulator. However, the project has not been free from challenges.

The biggest challenge involved the acquisition of data to drive the simulator. In particular, data on certain activities times (e.g., the movement times of crews), and on equipment failure and repair time was onerous to obtain in appropriate form and resolution. In some cases, the data was nonexistent and difficult to obtain (e.g., the movement times of crews on extremely rough terrain or in dense foliage). In other cases, although the technology was in place to collect the data, it was not being reported and/or archived properly due to lack of adequate resources. Finally, certain data that had been historically collected and archived was not at the right level of granularity for operations modeling (e.g., data that had been collected for financial reporting). Fortunately, many of these problems should be corrected in the near future since most of the technology required to record and archive good data for the simulator is already in place.

The other challenge is ongoing. The simulator will have a profound impact on the business of planning and conducting signal quality surveys. It will affect the way in which operations data is collected and archived (re last paragraph), it will change the way people model operations and model uncertainty (see Section 4.1), and it will generate results that will impact the way people make decisions. On the latter point, the simulation will enable decision makers to explicitly incorporate uncertainty into their decision making because simulation generates probability distributions estimates rather than using adjusted point estimates. Such changes are bound to take time to implement, perfect and integrate into the culture.

\section{ACKNOWLEDGEMENTS}

The authors would like to thank Peter Highnam and Ruven Brooks for their contributions to this work.

\section{REFERENCES}

Joines, J. A. and S. D. Roberts. 1994. Design of ObjectOriented Simulations in $\mathrm{C}++$. In Proceedings of the 
1994 Winter Simulation Confercnce Proceedings, ed. J. D. Tew, S. Manivannan, D. A. Sadowski, and A. F. Seila, 157-165. Institute of Electrical and Electronics Engineers, Piscataway, New Jersey.

Mesquite Software, Inc. 1994. CSIMI7 Users' Guide. Austin, Texas: Mesquite Software, Inc.

Shaw, M. and D. Garlan. 1996. Software Architecture: Perspectives on An Emerging Disciplinc. Saddle River, New Jersey: Prentice Hall.

Microsoft Corporation. 1995. Microsoft Foundation Class Library, Part One: Microsoft Visual C++, Version 4. Redmond, Washington: Microsoft Press.

\section{AUTHOR BIOGRAPHIES}

DOUGLAS J. MORRICE is an Associate Professor in the MSIS Department at The University of Texas at Austin. He received his undergraduate degree in Operations Research at Carleton University in Ottawa, Canada. He holds an M.S. and a Ph.D. in Operations Research and Industrial Engineering from Cornell University. His research interests include discrete event and qualitative simulation modeling and the statistical design and analysis of large scale simulation experiments. Dr. Morrice is a member INFORMS. He served as the Secretary for the INFORMS College on Simulation (1994-1996) and was Co-Editor of the Proceedings of the 1996 Winter Simulation Conference.

PETER W. MULLARKEY is a Research Scientist in the End-to-End Simulation Department at Schlumberger Austin Product Center - Research. He received his undergraduate degree in Civil Engineering at Worcester Polytechnic Institute in Worcester, Massachusetts. He holds a Ph.D. in Civil Engineering from CarnegieMellon University. Dr. Mullarkey has worked with Schlumberger doing research and development of knowledge-based decision support systems since 1985. Additionally, he worked on a two year project at Tandem Computers developing a state model-based diagnosis and maintenance system product.

ASTRID S. KENYON is a PhD student in the Department of Operations Research and Industrial Engineering at the University of Texas at Austin. She received her undergraduate degree in Engineering from Ecole Polytechnique Feminine in Sceaux, France. She holds an M.S. in Mechanical Engineering from the University of Texas at Austin. Her research interests include stochastic programming and multi-vehicle routing problems.

HERB SCHWETMAN is founder and president of Mesquite Software, Inc. Prior to founding Mesquite
Software in 1994, he was a Senior Member of the Technical Staff at MCC from 1984 until 1994. From 1972 until 1984, he was a Professor of Computer Sciences at Purdue University. He received his Ph.D. in Computer Science from The University of Texas at Austin in 1970. He has been involved in research into system modeling and simulation as applied to computer systems since 1968

JINGFANG ZHOU is currently an engineer at Schlumberger, Austin, Texas. Her research interests include Discrete Event Simulation, CAD/CAM, computational geometry, differential geometry, geographical information systems, scientific databases, and visualization. She received a B.S. and an M.S. in Naval Architecture and Marine Engineering from Huazhong University of Science and Technology, China, in 1985 and 1988, and a Ph.D in Computer Aided Geometric Design from MIT in 1995. 